\title{
Etiological Factors, Diagnoses, and Treatments of Halitosis: A Review update
}

\author{
Kabir $\mathrm{MH}^{1}$, Sarwar AFM² , Hossain $\mathrm{M}^{3}$, Ahmed I
}

\begin{abstract}
Halitosis is known as bad breath that emanates from the oral cavity either intra-oral and/or extra-oral origins with different nomenclature such as oral malodor, foetor ex ore, bad breath, foul smell or in Bengali durgondha. Fifty percent of people worldwide view themselves as having halitosis, with (85.0-90.0\%) of the etiology being intra-oral. Oral causes include basically tongue coating and other various causes like tooth decay, periodontitis, chronic gingivitis, oral infections, pericoronitis, calculus deposition, oral mucosal ulceration (bacterial and/or fungal), impacted food particles, orthodontic problems and poor oral hygiene practice. These sorts of foul smell of the oral cavity most often results from the bacterial degradation of oral organic substrates as volatile sulfur compounds (VSC). The source of halitosis may be related both to oral and systemic condition, but majority of cases are commonly related to oral factors. So far knowledge goes there have been a few studies evaluating the prevalence of bad mouth breath (BMB) in general population. The aim of this reviews are the classification of halitosis, assessment, diagnosis, intra-oral and systemic contributing factors, treatment, management and clinical application. [J Shaheed Suhrawardy Med Coll, 2013;5(2):106-110]
\end{abstract}

Key words: Halitosis, oral malodor, bad breath, assessment, classification, diagnosis, treatment

Recived: December 2012; Revised: March 2013; Accepted: May 2013

\subsection{Introduction}

Oral malodor or BMB is an unpleasant universal medicosocial problem ${ }^{1}$; therefore it has always been a factor of concern for society experienced by most individuals' results in transient discomfort ${ }^{2}$. At least $50.0 \%$ of the peoples are suffering from chronic oral malodor ${ }^{3}$. The air from the mouth of chronic malodor sufferers are tainted with compounds such as hydrogen sulfide (H2S), methylmercaptan $(\mathrm{CH} 3 \mathrm{SH})$, dimethylsulfide [(CH3)2S] and organic acids, which produce a series of foul air that is very offensive to the people in their vicinity ${ }^{4-9}$. While dental diseases have been strongly associated with such condition, there is considerable evidence that dentally healthy individuals can exhibit significant levels of halitosis. Proteolytic activity by microorganisms over the tongue and teeth results the foul-smelling compounds, and is the most common cause of such problems ${ }^{10}$. This foetor ex ore can have major detrimental social activities for the sufferer and directly impact on normal social communication. Most cases of BMB are mainly of intraoral origin ${ }^{11}$. It is a common complaint of up to one-third of the general population and arises from a variety of sources including the sinuses, gastrointestinal tract, ingested food, lungs, and most frequently in the oral cavity. The substances of malodorous are most commonly related with by bacterial metabolic degradation and occur on oral surfaces, in periodontal pockets, and especially on the dorsum of the tongue ${ }^{12}$. In this review the different etiological factors, diagnosis and treatment were reviewed.

\section{History}

The prophet Hazrat Mohammad (PBUH) was said to have thrown congregant from the mosque for having the smell of garlic on his breath. And it is known that bad breath has been recorded in the literature for thousands of year. Such problems were discussed at length in the Jewish Talmud as

1. Dr. Md. Humayun Kabir, Associate Professor, Department of Dentistry, Dental Unit, Shaheed Suhrawardy Medical College, Dhaka

2. Dr. A.F.M. Sarwar, Assistant Professor, Children, Preventive and Community Dentistry, Dhaka Dental College, Dhaka

3. Dr. Motaher Hossain, Proprietor and Dental Surgeon, Prime Dental Clinic, Dhaka

4. Dr. Ikramul Ahmed, Assistant Professor, Children, Preventive and Community Dentistry, Dhaka Dental College, Dhaka

Correspondence

Dr. A.F.M. Sarwar, Assistant Professor, Children, Preventive and Community Dentistry, Dhaka Dental College, Dhaka, Bangladesh; Email: drsarwarbd@gmail.com; Cell: +8801911900702

Conflict of Interest: None

Financial support: None

Contribution to Authors: All authors contribute during the preparation of article. 
well as by Greek and Roman writers ${ }^{19}$. In the Bible, the book of Genesis mentions ladanum (mastic), a resin derived from the Pistacia lentiscus tree, which had been used in Mediterranean countries for breath freshening for thousands of years. Chewing of natural products for breath freshening has long been practiced worldwide, such as cloves (Iraq), parsley (Italy), anise seeds (Far East), cinnamon (Brazil), and guava peels (Thailand) and eggshell (China) ${ }^{14,19-20}$. Mouthwashes containing flavored elements like menthol, eucalyptol and methyl salicylate are also widely used. Knowledge and written reference to this condition date back to ancient cultures. References were found in papyrus manuscripts dating back to $1550 \mathrm{BC}^{14}$. Since the 1960s preeminent researcher in this field has been Dr. Joseph Tonzetich of the University of British Columbia. Among others findings Tonzetich and co workers established that oral mal odor is associated with the presence of VSCs compounds primarily $\mathrm{H} 2 \mathrm{~S}$ and $\mathrm{CH}_{3 \mathrm{SH}^{19}}$. Hippocrates $(460-400$ B.C.) the father of medicine $^{21}$ had developed a mouthwash of unadulterated wine, anise, dill seed and myrtle. Maccius Platus (254-184 B.C.) a Roman dramatist who was sufficiently offended by his wife's halitosis to find it just cause for infidelity. In 19th century Joseph Howe, a physician, introduced his well-written and informative book. He believed that halitosis was the result of sulphuretted hydrogen, which is found in great abundance in the intestinal canal as well as in decayed teeth, dead teeth and inflamed gums ${ }^{22}$. For this reason they used mechanisms to hide halitosis, such as perfumed tablets, chewing leaves and stalks of plants. In Antiquity, having a fragrant breath meant exhaling the sweetness of life and asserting the purity of a person's soul. During Christianity, the devil's supreme malignant odor smelled of sulfur, and it was presumed that sins produced a more or less bad smell. This association is complex, as the predominant smell in halitosis is often sulfur. A treaty in Islamic literature from the year 850 talked about dentistry, referring to the treatment of fetid breath, and recommended the use of siwak when breath had changed or at any time when getting out of bed. The Hindus consider the mouth as the body's entry door and, therefore, insist that it be kept clean, mainly before prayers. The ritual is not limited to teeth brushing, but includes scraping the tongue with a special instrument and using mouthwash. Buddhist Monks in Japan also recommended teeth brushing and tongue scraping before the first morning prayers. Nowadays, it is known that multiple causative factors can produce halitosis, making it impossible to indicate one single treatment; after the diagnosis, the initial behavior adopted is the removal of possible causes ${ }^{2}$. The universal medical term "halitosis" used since 1930 comes from "Halitus" meaning "breath", and "-osis" meaning "chronic disorder". In the era of "Renaissance", Laurent Joubert (1529-1582), the medical doctor quantifying oral malodor: organoleptic measurement, gas chromatography (GC), and sulfide monitoring ${ }^{23}$, Spoon test, the dental floss odor test and BANA test.

\section{Types of Halitosis}

Halitosis is a widespread condition may be an important factor in social communication, and therefore may be the origin of concern not only for a possible health condition but also for frequent psychological alterations, leading to social and personal isolation ${ }^{13}$. It is the common term used to describe any disagreeable odor in expired air, regardless of whether the odorous substances originate from oral or non-oral sources ${ }^{14-15}$ and that should be treated by the dental professional ${ }^{16}$. This can be classified into three groups of genuine halitosis (the breath malodour is a real problem that can be easily diagnosed either by organoleptic or by physic-chemical means), pseudohalitosis (the oral malodour does not exist, but the patient believes that he or she has it) and halitophobia (after successful treatment for either genuine halitosis or pseudo-halitosis the patient still believes that he or she has halitosis, then the diagnosis is termed "halitophobia") ${ }^{17}$ which has a multifactorial etiology involving extrinsic and intrinsic factors ${ }^{18}$.

\section{Etiological Factors}

The etiology of oral malodor is multifactorial. Sulfur and Non-sulfur-containing compounds such as cadaverine, putrescine, indole, skatole, and volatile fatty acids such as butyric or propionic acid have also been implicated in the foul smell of oral malodour ${ }^{9}$ that can be segmented into seven main origin like Mouth \& Tongue, Nasal, Nasopharyngeal, Sinus \& Oro pharyngeal, Xerostomia induced, Primary Lower Respiratory Tract \& Lung, Systemic diseases \& disorders, GI Tract diseases \& disorders and Odiferous food, fluid and medication consumptions $^{24}$. Most of these compounds result from the proteolytic degradation by oral microorganisms of peptides present in saliva, shed epithelium, food debris, gingival crevicular fluid (GCF), interdental plaque, postnasal drip, and blood. In particular, gram- negative, anaerobic bacteria possess such proteolytic activity. Proteolysis of proteins, peptides, amino acids, free thiol groups and volatile sulphides $^{9}$ are closely associated with disorders such as periodontal diseases, poor oral hygiene, tongue coating, dry mouth (xerostomia), dental caries, oral carcinoma, and several forms of stomatitis ${ }^{25}$. Predisposing factors include poor oral hygiene, gingival and periodontal disease, disorders of the oral mucosa, reduced salivary flow and wearing of dental appliances ${ }^{13}$, may develop such problems and other causes are as renal impairment is normally a result of a chronic glomerulonephritis, which damage the glomerular function, leading to an increased urea level in the blood. Breathed air is described as ammonium-like breath and generally is accompanied by complaints of dysgeusia (salty taste). Diabetes Mellitus can result in accumulation of ketone bodies, which are breathed out producing a very characteristic halitus, moreover, diabetes causes dry mouth due to decrease in saliva production. Trimethylaminuria or "fish odor syndrome" is a genetic metabolic disorder ${ }^{26}$ characterized by a failure in the oxidation route from trimethylamine (TMA) to 
trimethylamine N-oxide (TMA-O) in the liver. High levels of TMA in urine and others body fluids confer that typical unpleasant, intermittent characteristic fishy odor to the breath. Radiation therapy, Sjogren's syndrome, and certain types of carcinomas such as leukemia can also contribute to oral malodour ${ }^{9,27}$. Most of the salivary proteins available in the oral cavity are glycoproteins, whose carbohydrate components could generally interfere with their proteolysis $^{25}$. Dorsum of the tongue is the primarily identified as the principal location for the production of oral malodor because the tongue is a haven for the growth of microbial. The proteolytic, anaerobic bacteria that reside on the tongue play an essential part in the development of oral malodor and the specific character of breath odors are A "rotten eggs" smell is indicative of VSCs, A sweet smell, as that of "dead mice" has been associated with liver insufficiency; besides VSCs, aliphatic acids (butyric, isobutyric, propionic) accumulate, The smell of "rotten apples" has been associated with unbalanced insulindependant diabetes, which leads to the accumulation of ketones and A "fish breath" can suggest kidney insufficiency characterized by uremia and accumulation of dimethylamine and trimethylamine ${ }^{9}$. Anaerobic bacteria, oxygen depletion, alkaline $\mathrm{pH}$ and sulfur-containing substrates are some of the requirements for oral malodor to occur. Management of oral malodor is therefore dependent on diagnosing the foul breath as physiological or pathological and therapy should then be appropriately and specifically directed to the cause of the oral malodor to ameliorate or totally eliminate the condition.

\section{Table 1: Etiological factors for BMB ${ }^{11}$}

\section{Intra oral}

Intra oral bacteria: Tongue colonization, Chronic periodontitis

Acute oral infection: ANUG, Pericoronitis, Acute herpetic gingivostomatitis

\section{Extra oral}

Nasal and pharyngeal infection: Post nasal drip, Chronic sinusitis, Foreign body

Respiratory condition: Chronic bronchitis, Bronchial carcinoma

GI condition: esophageal reflux, pyloric stenosis

Metabolic condition: diabetic ketoacidosis, renal failure, hepatic failure, trimethylaminuria

\section{Psychogenic}

Halitophobia

\section{Transient}

Dietary: Garlic, Onion, Spicy food

Tobacco: Chewing, Smoking, Gul

\section{Diagnosis}

There are some methods of quantifying oral malodour like organoleptic measurement, gas chromatography (GC), and sulphide monitoring. Organoleptic measurement: A subjective test scored on the basis of the examiner's perception of a subject's oral malodour is to be assessing by consensus on using a scale ranging from 0 to 5 .

Gas chromatography (GC): GC is considered the gold standard for measuring oral malodour since it is specific for VSCs, the main cause of oral malodour.

Sulphide monitoring: Sulphide monitors analyse for total sulphur content of the subject's mouth air. Although compact sulphide monitors are inexpensive, portable, and easy to use, most of them are not able to distinguish among the $\mathrm{VSCs}^{17}$.

Spoon test $^{28}$ : It assesses odor from the dorsum of the posterior tongue. A plastic spoon is used to scrape and scoop material from the back region of the tongue dorsum. The spoon odor is evaluated after five seconds at a distance of approximately $5 \mathrm{~cm}$ from the examiner's nose.

The dental floss odor test ${ }^{29}$ : It determines the presence of interdental plaque odor. Unwaxed floss is passed through inter proximal contacts of the posterior teeth and the examiner assesses the odor by smelling the floss at a distance of approximately $3 \mathrm{~cm}$.

The saliva odor test: subject expectorates approximately 1 to 2 milliliters of saliva into a Petri dish. The dish is covered immediately, incubated at $370 \mathrm{C} 30$ for five minutes and is then presented for odor evaluation at a distance of $4 \mathrm{~cm}$ from the examiner's nose. A scale commonly used in malodor research is the $0-5$ intensity scale is used and this organoleptic scale, 0 indicates a concentration of odorant that is below a threshold, and 5 indicates concentrations that are extremely strong. The scores are 0 -absence of odor, 1-questionable odor, 2-slight malodor, 3-moderate malodor, 4-strong malodor, 5-severe malodor.

BANA test $^{31}$ (benzoyl-arginine-naphthylamide) is used chairside to determine the proteolytic activity of certain oral anaerobes that contribute to oral malodor. WTCI (Winkel Tongue Coating Index) divided the dorsal surface of the tongue into sextants, 3 in the posterior and 3 in the anterior part of the tongue. Scoring criteria is 0 -no coating, 1-presence of light coating, 2-presence of a distinct coating. The resulting WTCI is obtained by adding all 6 scores9. The Miyazaki Index was used to assess the efficacy of various treatment procedures in reducing oral malodor by reducing the coating on the tongue. A single score for the entire tongue, as well as score per area (anterior and posterior to the sulcus terminalis, each region further divided into left and right sides) is given. The 
scores ranged from 0 to 3 categorized 0 -no coating, $1-<$ $1 / 3,2-<2 / 3$, and $3->2 / 3$ of the surface coated $)^{32-33}$.

Personal assessment of oral malodour: Self-AssessmentLick wrist, wait $5 \mathrm{~min}$, then smell, Counterpart Assessment- Wife, Husband, Partner, Fresh Breath Detective- Uses Cotton Swabs and Oral Judges.

\section{Treatment of Oral Malodor}

Morning breath from the oral cavity can be controlled by proper oral hygiene ${ }^{34}$ practice. It is known that halitosis affects a person's daily life negatively. There are several methods to determine halitosis. It can be treated if its etiology can be detected appropriately through proper detailed clinical examination ${ }^{35}$. The ultimate goal of treatment for oral malodor should be directed at eliminating or at least reducing the causative microorganisms and associated substrates. If the microorganisms quantity and plaque maturity are controlled, then their capabilities to produce VSCs is greatly reduced. The substrate available for metabolic breakdown should likewise be eliminated or reduced with good oral hygiene and control of periodontal inflammatory disease. Following general treatment strategies can be applied: mechanical reduction of intraoral nutrients and microorganisms: tongue cleaning, inter dental cleaning and toothbrushing are essential mechanical means of dental plaque control; chemical reduction of oral microbial load: Mouthwashes have been used as chemical approach to combat oral malodor. Antibacterial components in oral rinses such as cetylpyridinium chloride (CPC), chlorhexidine, triclosan, essential oils, quaternary ammonium compounds, benzalkonium chloride and hydrogen peroxide have been considered along with mechanical approaches to reduce oral malodour; rendering malodorous gases non-volatile: zinc salt containing mouthwashes, baking soda dentifrices and chewing gum formulated with antibacterial agents and tea extracts like epigallocatchin and masking the malodor: mouth sprays and lozenges containing volatiles with a pleasant odor ${ }^{9}$.

\section{Tips for the patients who are suffering from BMB}

Taking a some minutes every day to clean mouth thoroughly, cleaning of the tongue, wet whistle, combat post nasal drip and visiting doctor for undiagnosed extra oral disease ${ }^{23}$.

\section{Conclusion}

Breath malodor has important socioeconomic consequences. It can reveal important diseases. However, majority of cases of oral malodour are due to bacterial proteolytic activity in the mouth; therefore, dentists can assist patients to control the condition by providing fullmouth disinfection and treatment modality for oral problems by providing suggestions on oral hygiene, or referring them for medical opinion when a non-oral cause is assumed. A proper diagnosis and determination of the etiology allows initiation of proper etiologic treatment. It is suggested that lack of knowledge about how to prevent halitosis permits its occurrence and limits quality of life. These problems could easily be solved through health education, as the causes of halitosis are basically concentrated in the mouth.

\section{References}

1. Eldarrat A, Alkhabuli J, Malik A. The Prevalence of Self-Reported Halitosis and Oral Hygiene Practices among Libyan Students and Office Workers. Libyan Journal of Medicine 2008; 3(4): 170-176

2. Elias MS, Ferriani MDGC. Historical and social aspects of halitosis. Revista latino-americana de enfermagem 2006;14(5);821-823

3. Armstrong BL, Sensat ML, Stoltenberg JL. Halitosis: a review of current literature. American Dental Hygienists Association 2010;84(2), $65-74$

4. Miyazaki H, Sakao S, Katoh Y, Takehara T. Correlation between volatile sulphur compounds and certain oral health measurements in the general population. Journal of Periodontology 1995;66(8):679-84

5. Soder B, Johansson B, Söder PO. The relation between foetor ex ore, oral hygiene and periodontal disease. Swedish Dental Journal 2000;24(3):73-82

6. Bornstein MM, Kislig K, Hoti BB, Seemann R, Lussi A. Prevalence of halitosis in the population of the city of Bern, Switzerland: a study comparing self-reported and clinical data. European Journal of Oral Sciences 2009;117(3):261-7

7. Yokoyama S, Ohnuki M, Shinada K, Ueno M, Wright FA, Kawaguchi Y. Oral malodor and related factors in Japanese senior high school students. Journal of School Health 2010;80(7):346-52.

8. Loesche WJ, Kazor C. Microbiology and treatment of halitosis. Periodontology 2000. 2002;28:256-79

9. Porter SR, Scully C. Oral malodour (halitosis). BMJ 2006;23;333(7569):632-635.

10. Nalini Saini1, Puneet Ajwani, Kulmeet Kaur and Amandeep Kumar. Oral Malodor: A Common Oral Problem. J Bioengineer \& Biomedical Sci 2011, 2:1 doi:10.4172/2155-9538.1000108.

11. Anne Bosy. Oral malodour: philosophical and practical aspects Canadian Dental Association/L'Association Dentaire Canadienne Journal March/Mars 1997;63(3)

12. Francis J. Hughesa, Rod McNabb. Oral malodour - a review. Archives of oral biology 53, Suppl. 1 (2008) S1-S7. Available at the journal homepage: www.intl.elsevierhealth.com/journals/arob

13. C. E. Kazor, P. M. Mitchell, A. M. Lee, L. N. Stokes, W. J. Loesche, F. E. Dewhirst, and B. J. Paster. Diversity of Bacterial Populations on the Tongue Dorsa of Patients with Halitosis and Healthy Patients. journal of clinical microbiology, Feb. 2003;41(2):558-563

14. Rajiv Saini, Santosh Saini and Sugandha R Saini. Halitosis: An oral microbial faction. 2010;1(4):36-39

15. Naila Aparecida de Godoi Machado; Paulinne Junqueira Silva Andresen Strini; Mario Olavo Pena Carneiro; Roberto Bernardino Júnior; Alfredo Júlio Fernandes Neto. Halitosis: a review of basic principlesBraz J Oral Sci. 7(26):1627-1630

16. Basavaraj P and Nitin Khuller. Halitosis: A Review. Indian J Stomatol 2011;2(3):183-86.

17. ADA Council on Scientific Affairs. Oral malodor. J Am Dent Assoc. 2003;134(2):209-214

18. Sanz M, Roldán S, Herrera D. Fundamentals of Breath Malodour. J Contemp Dent Pract 2001 Nov;(2)4: 001-017

19. P. Youngnak-Piboonratanakit and T. Vachirarojpisan. Prevalence of Self-Perceived Oral Malodor in a Group of Thai Dental Patients. Journal of Dentistry, Tehran University of Medical Sciences, Tehran, Iran 
2010;7(4):196-204

20. Mel Rosenberg. Clinical assessment of breath: Current Concepts. JADA April 1996;127:475-482

21. PPC Lee, WY Mak and P Newsome. The aetiology and treatment of oral halitosis: an update. Hong Kong Med J 2004;10(6):414-8

22. Roberta Ratcliff. Current Concepts in the Cause and Treatment of Halitosis.Practicle Hygiene 1997:47-53

23. Anoop Kapoor, Vishakha Grover, Ranjan Malhotra, Supreet Kaur and Kishan Singh. Halitosis - Revisited Indian Journal of Dental Sciences. December 2011;3(5):102-111

24. Dr. Rafif Tayara and Dr. Riad Bacho. Oral Medicine. Smile Dental Journal 2009;4(2):10-12

25. Kristy Menage Bernie. The Breath of Health: Considerations for Oral Malodor Management. CDHA Journal 20(2):13-19 [Web Site: http://www.cdha.org/downloads/ce_courses/homestudy_breath.pdf 26. Sachiko Takehara, Masaki Yanagishita, Katarzyna Anna PodymaInoue, Masayuki Ueno, Kayoko Shinada and Yoko Kawaguchi. Relationship between Oral Malodor and Glycosylated Salivary Proteins. J Med Dent Sci 2010; 57:25-33

27. S. C. Mitchell and R L. Smith. Trimethylaminuria: the fish malodor syndrome. Drug metabolism and disposition 2001;29(4):517-521
28. José Roberto Cortelli, Mônica Dourado Silva Barbosa and Miriam Ardigó Westphal. Braz Oral Res 2008;22(Spec Iss 1):44-54

29. Ongole R, Shenoy N. Halitosis: Much beyond oral malodor. Kathmandu University Medical Journal 2010;8(2-30): 269-275

30. Suvarna H Patil, Anita Kulloli, Minal Kella. Unmasking Oral Malodor: A Review. People's J Scientific Res 2012;5(1):61-68

31. Quirynen M, Zhao H, Avontroodt P, Soers C, Pauwels M, Coucke W, van Steenberghe D. A salivary incubation test for evaluation of oral malodor: a pilot study. J Periodontol. 2003 Jul;74(7):937-44

32. A. Kozlovskyl, D. Gordon', Gelernter, WJ. Loesche, and M. Rosenberg. Correlation between the BANA Test and Oral Malodor Parameters. J Dent Res May 1994;73(5):1036-1042

33. PK Pratibha,, KM Bhat, and GS Bhat. Oral Malodor: A Review of the Literature Journal of Dental Hygiene, An Electronic Reprint From July 2006;80(3):1-10

34. S R Porter, C Scully. Oral malodour (halitosis). BMJ 2006;333:632-5

35. Tonzetich J, Ng SK. Reduction of malodor by oral cleansing procedures. Oral Surg Oral Med Oral Pathol. 1976 Aug;42(2):172-81

36. Bahadir Ugur Aylikci and Hakan Çolak. Halitosis: From diagnosis to management. 2013;4(1):14-23 